\title{
Cognitive impairment in generalized anxiety disorder revealed by event-related potential N270
}

This article was published in the following Dove Press journal:

Neuropsychiatric Disease and Treatment

3 June 2015

Number of times this article has been viewed

\author{
Yingxue Yang ${ }^{1,2}$ \\ Xiating Zhang ${ }^{1,2}$ \\ Yu Zhu ${ }^{1,2}$ \\ Yakang Dai ${ }^{3}$ \\ Ting Liu ${ }^{3}$ \\ Yuping Wang ${ }^{1,2}$
}

'Department of Neurology, Xuanwu Hospital, Capital Medical University, ${ }^{2}$ Beijing Key Laboratory of Neuromodulation, Beijing, People's Republic of China; ${ }^{3}$ Suzhou Institute of Biomedical Engineering and Technology, Chinese Academy of Sciences, Suzhou, People's Republic of China
Correspondence: Yuping Wang Department of Neurology, Xuanwu Hospital, Capital Medical University, No 45 Changchun Street, Xicheng District, Beijing 100053, People's Republic of China

Tel +861083198273

Fax +861083157841

Email wangyyping@yeah.net
Background: Cognitive function in anxiety disorders has been the subject of limited investigation, especially in generalized anxiety disorder (GAD). The purpose of this study was to investigate the cognitive function in subjects with GAD using mismatch-triggered negativity N270.

Methods: Fifteen medication-free patients with a DSM-IV diagnosis of GAD, and 15 wellmatched healthy controls performed a dual-feature delayed matching task while event-related potentials were recorded from their scalp.

Results: The GAD group was characterized by the decreased N270 amplitude in the left hemisphere. The smaller N270 amplitude was associated with greater symptoms of anxiety and depression.

Conclusion: Since N270 is thought to index cognitive function in different domains, including attention and memory, our results suggest that individuals with GAD have an impaired cognitive function, particularly in selective attention and working memory. These cognitive deficits may have clinical significance in subjects with GAD and should be considered in treatment planning.

Keywords: generalized anxiety disorder, N270, cognitive function, selective attention, working memory

\section{Introduction}

Generalized anxiety disorder (GAD) is a serious and common psychiatric disorder characterized by excessive worry and anxiety, and associated with impaired social and occupational functioning. Anxiety disorders, including obsessive-compulsive disorder and post-traumatic stress disorder, are associated with poor neuropsychological performance in attention and memory. ${ }^{1,2}$ However, relatively little is known about the nature of the cognitive deficits in subjects with GAD. In a recent study, young subjects with GAD performed worse on executive and nonverbal memory tasks compared to controls. ${ }^{3,4}$ Research in elderly subjects with GAD has reported associated cognitive impairments in working memory and inhibition. ${ }^{5}$ However, results are frequently inconsistent and several studies have failed to find any major cognitive dysfunction in subjects with GAD. ${ }^{6,7}$ Eventrelated potentials (ERPs) reveal the cognitive function of the brain with high temporal resolution. Previous studies have suggested that N270, an endogenous ERP component, is an important and sensitive component used for the evaluation of the cognitive status of patients with Parkinson's disease, major depression, and transient ischemic attack. ${ }^{8-10}$ N270 is a negative deflection with a frontocentral scalp distribution that peaks at around $270 \mathrm{~ms}$ and is elicited by mismatch trials. ${ }^{11}$ More specifically, it is evoked by sequentially presented mismatches but is absent for simultaneously presented mismatches. ${ }^{12}$ In addition, the amplitude of $\mathrm{N} 270$ can be modulated by attention. ${ }^{13}$ Collectively, these results suggest that N270 may index multiple cognitive processes, including working memory 
and attention. Source analyses have also indicated that N270 originates in the dorsolateral prefrontal cortex and anterior cingulated cortex. ${ }^{14,15}$ Because these regions are also implicated in the pathophysiology of GAD,${ }^{16}$ study of this component may be ideal for identifying the specific nature of cognitive processing deficits in subjects with GAD.

The current study aimed to compare electrophysiological (N270) and behavioral data between individuals with GAD and healthy controls using a delayed matching-to-sample task. Based on previous studies, ${ }^{3,5,16}$ we predicted that reduced electrophysiological activation in subjects with GAD would be suggestive of deficits in basic cognitive processing.

\section{Materials and methods Subjects}

Fifteen right-handed, medication-free patients (eight women), diagnosed with GAD according to DSM-IV criteria were recruited at the outpatient ward of Xuanwu Hospital, Capital Medical University, Beijing, People's Republic of China. Patients with comorbid Axis I disorders, including current major depressive episodes, alcohol or substance abuse, history of traumatic head injury, and neurological or medical illnesses, were excluded from the study. Fifteen right-handed healthy control subjects (eight women), matched for age and education, were recruited from the local community. These control subjects had no DSM-IV Axis I disorders, alcohol or substance abuse, head trauma, or neurological or major medical illness. The Hamilton Anxiety Scale (HAMA), ${ }^{17}$ Hamilton Depression Scale (HAMD), ${ }^{18}$ and State-Trait Anxiety Inventory (STAI) ${ }^{19}$ were administered to all the subjects. The demographic and clinical characteristics of the subjects are shown in Table 1. The subjects well matched on demographic data but differed on symptom ratings.

This study was approved by the Ethics Committee of Xuanwu Hospital, and written informed consent was obtained from all the subjects before participation.

\section{Experimental task}

A dual-feature delayed matching paradigm similar to that used by Wang et $\mathrm{al}^{20}$ was used in this experiment. Each stimulus was of one of four colors (red, yellow, green, or white) and one of ten shapes (triangle, quadrangle, pentagon, hexagon, rhombus, arrow, crisscross, round, pentacle, or ellipse). A stimulus system (STIM; Neurosoft Inc., Sterling, USA) was employed for controlling stimuli presentation. For each trial, two stimuli of any one pair (S1 and S2) were sequentially presented for the duration of $500 \mathrm{~ms}$ each with
Table I Demographic and clinical characteristics of subjects (mean \pm SD)

\begin{tabular}{lll}
\hline & Controls & GAD \\
\hline Age (years) & $29.4 \pm 5.3$ & $31.1 \pm 5.7$ \\
Education (years) & $14.9 \pm 3.3$ & $14.0 \pm 3.4$ \\
HAMA & $1.3 \pm 1.4$ & $20.5 \pm 3.0^{\mathrm{a}}$ \\
HAMD & $0.6 \pm 1.2$ & $13.6 \pm 3.4^{\mathrm{a}}$ \\
STAI & $62.2 \pm 9.8$ & $105.8 \pm 21.6^{\mathrm{a}}$ \\
\hline
\end{tabular}

Notes: aSignificantly different from the control group $(P<0.05)$. Controls are healthy subjects.

Abbreviations: SD, standard deviation; GAD, generalized anxiety disorder; HAMA, Hamilton Anxiety Scale; HAMD, Hamilton Depression Scale; STAI, State-Trait Anxiety Inventory.

an interstimulus interval of $200 \mathrm{~ms}$. The intertrial interval was 5 seconds. The stimulus pairs were divided into four conditions of equal probability: $\mathrm{C}-\mathrm{S}-$, color same $(\mathrm{C}-)$, shape same $(\mathrm{S}-)$ (match); $\mathrm{C}-\mathrm{S}+$, color same $(\mathrm{C}-)$, shape different $(\mathrm{S}+)$ (task-irrelevant mismatch); $\mathrm{C}+\mathrm{S}-$, color different $(\mathrm{C}+)$, shape same $(\mathrm{S}-)$ (task-relevant mismatch); and $\mathrm{C}+\mathrm{S}+$, color different $(\mathrm{C}+)$, shape different $(\mathrm{S}+)$ (conjunction mismatches). The visual stimuli were presented at the center of a computer-controlled monitor screen to each participant. The four conditions of stimulus pairs were randomly presented in sequence and had equal probability. The averaged visual angle of $\mathrm{S} 1$ and $\mathrm{S} 2$ was adjusted to $2.1^{\circ}$.

Following electrode application, subjects sat in a comfortable chair and fixated at the center of the screen in front of them. For the experiment, subjects were asked to decide whether the color of S2 was identical to that of the previously presented S1 and to ignore its shape: same color, left button press, and different color, right button press on a push pad. They were instructed to respond as quickly and accurately as possible, and the response hands were counterbalanced in the second half of the test in each subject. Because patients with GAD have difficulty focusing on tasks for a long time, other tests such as selective attention to shape were not conducted.

\section{ERP recordings}

Electroencephalography (EEG) was recorded from 20 scalp positions (FP1, FP2, F7, F8, F3, F4, FZ, T3, T4, T5, T6, C3, $\mathrm{C} 4, \mathrm{CZ}, \mathrm{P} 3, \mathrm{P} 4, \mathrm{PZ}, \mathrm{O} 1, \mathrm{O} 2, \mathrm{OZ})$ referenced to the nose using $\mathrm{Ag} / \mathrm{AgCl}$ electrodes. Vertical and horizontal electrooculograms were recorded by electrodes situated above and below the left eye, and on the outer canthi of both eyes, respectively. EEG data were amplified with a band pass of $0.1-330 \mathrm{~Hz}$ and sampled at 1,000 Hz. ERPs were averaged separately for each condition and digitally filtered with a band pass of $0.1-40 \mathrm{~Hz}$. The averaging epoch was $800 \mathrm{~ms}$; the first $200 \mathrm{~ms}$ 
before the $\mathrm{S} 2$ onset served as the baseline. Trials containing incorrect responses, electrooculogram artifacts, or amplifier saturation were rejected. More than 30 trials were averaged in each condition for each subject.

\section{Statistical analysis}

All statistical analyses were conducted using SPSS (Version 16.0). Group differences in demographic and clinical variables were examined using the independent-group $t$-test. In this study, the $\mathrm{N} 270$ component was most prominent at F3, F4, C3, and C4 electrodes, whereas P300 was most prominent at $\mathrm{P} 3$ and $\mathrm{P} 4$ electrodes; the amplitudes and peak latencies of the two components were thus measured at these respective electrodes. Based on previous studies and the grand-averaged results of this study, mean N270 amplitudes were measured in all four conditions in the time window of 270-290 ms after S2 onset. Mean P300 amplitudes were measured in the time window of 320-370 ms after S2 in the match condition only. Mean N270 amplitudes and peak latencies were submitted to a repeated measures analysis of variance (ANOVA) with the two groups (GAD and control) as the between-group factor and the conditions (four levels: match, task-irrelevant mismatch, task-relevant mismatch, and conjunction mismatches) and selected electrodes (F3, F4, C3, and C4) as within-subject factors. A two-way ANOVA was employed to analyze P300 mean amplitudes and peak latencies (the group factor: GAD, control; the electrode factor: P3, P4). Behavioral measures of correct response rate (CR) and reaction time (RT) were analyzed using a repeated measure ANOVA. The Greenhouse-Geisser correction and post hoc comparisons were conducted when appropriate. Finally, Pearson's correlation was utilized to examine the relationship between symptom measures and N270 features.

\section{Results}

\section{Behavioral data}

Behavioral CR and RT data are summarized in Table 2. There was no difference in $\mathrm{CR}(F[1,28]=2.066, P>0.05)$ or RT $(F[1,28]=3.756, P>0.05)$ between the two groups. RTs for both groups were significantly quicker in the match condition than in the task-relevant mismatch condition $(P<0.001)$.

\section{ERP data}

In the match condition, a P300 component was evoked by S2. In the mismatch condition, another ERP component, the N270, was elicited, followed by a P300 component. Grand-averaged ERP waveforms for the two groups are shown in Figure 1.
Table 2 CR and RT of the two groups in each of the four conditions (mean $\pm \mathrm{SD}$ )

\begin{tabular}{lll}
\hline & Controls & GAD \\
\hline CR $(\%)$ & & \\
Match & $98.7 \pm 2.1$ & $99.2 \pm 1.5$ \\
Task-irrelevant mismatch & $97.2 \pm 3.0$ & $98.8 \pm 1.9$ \\
Task-relevant mismatch & $98.0 \pm 2.4$ & $98.5 \pm 1.8$ \\
Conjunction mismatches & $98.7 \pm 2.1$ & $99.0 \pm 1.8$ \\
RT (ms) & & \\
Match & $544.3 \pm 120.7$ & $620.8 \pm 107.5$ \\
Task-irrelevant mismatch & $572.7 \pm 116.2$ & $627.9 \pm 105.7$ \\
Task-relevant mismatch & $597.3 \pm 111.7^{\mathrm{a}}$ & $673.7 \pm 102.5^{\mathrm{a}}$ \\
Conjunction mismatches & $584.9 \pm 120.6$ & $643.7 \pm 101.3$ \\
\hline
\end{tabular}

Notes: aSignificantly different from match condition in each group $(P<0.001)$. Controls are healthy subjects.

Abbreviations: CR, correct response rate; RT, reaction time; SD, standard deviation; GAD, generalized anxiety disorder.

Two patients showed no N270 response, and their data were excluded for statistical analysis of latency.

Between $270 \mathrm{~ms}$ and $290 \mathrm{~ms}$, an N270 was elicited in all mismatch conditions and by both groups. Controls showed a greater amplitude of N270 in task-relevant and conjunction mismatches conditions than did patients with GAD (Table 3, Figure 1B). There was a significant main effect of condition on N270 amplitude $(F[2.208,59.625]=14.566, P<0.001)$, and a post hoc analysis showed that the N270 amplitude elicited in the match condition was significantly smaller than those of the mismatch condition $(P<0.05)$. There was a significant three-way interaction between condition, electrode, and group $(F[9,243]=1.968, P<0.05)$. Post hoc tests revealed that in the task-relevant mismatch condition, the mean N270 amplitude was significantly smaller in the GAD group compared to the control group at electrode $\mathrm{F} 3(P<0.01)$, while in the conjunction mismatches condition, the N270 amplitude was significantly smaller in the GAD group at electrodes F3 and C3 $(P<0.01)$ compared to the control group. There was no significant group difference in N270 amplitude in the task-irrelevant mismatch condition. The peak latency of N270 did not differ significantly between the two groups $(F[1,26]=2.114, P>0.05)$ (Table 4).

The P300 mean amplitude $(F[1,28]=0.783, P>0.05)$ and peak latency $(F[1,28]=1.288, P>0.05)$ did not differ significantly between the two groups (Table 5 ).

\section{Correlation findings}

In order to study the relationship between the N270 response and the patients' clinical symptoms, Pearson's correlation coefficients between the N270 amplitude (recorded at four electrodes), and the HAMA score, HAMD score, and STAI 
A
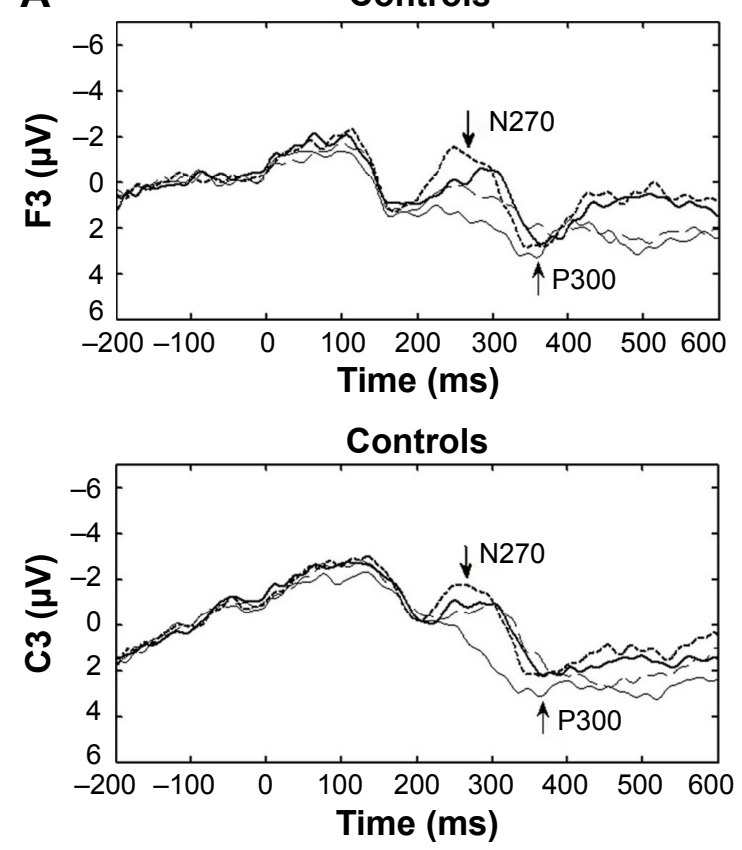

$B$
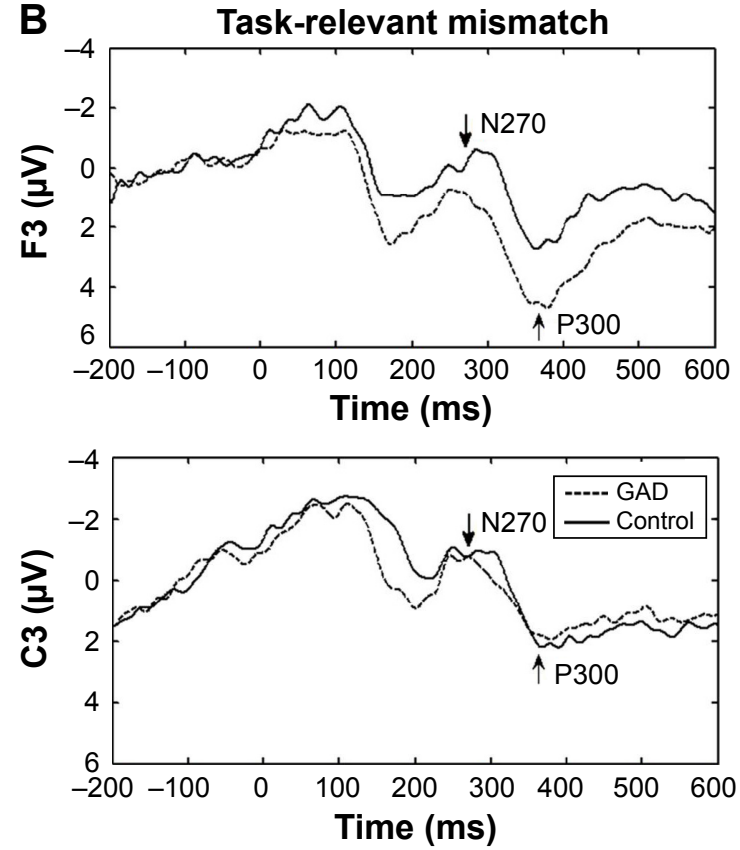

Controls

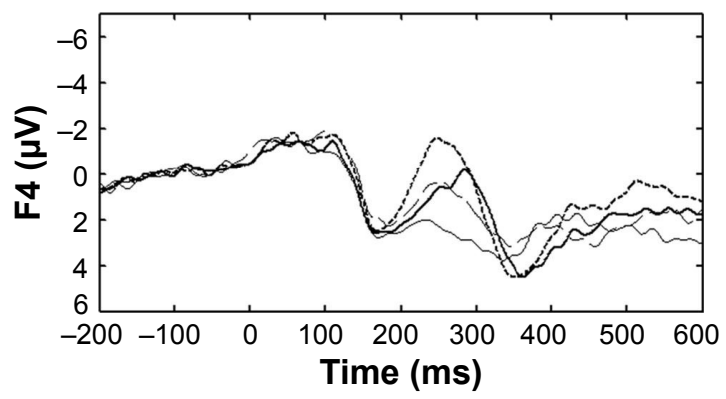

Controls
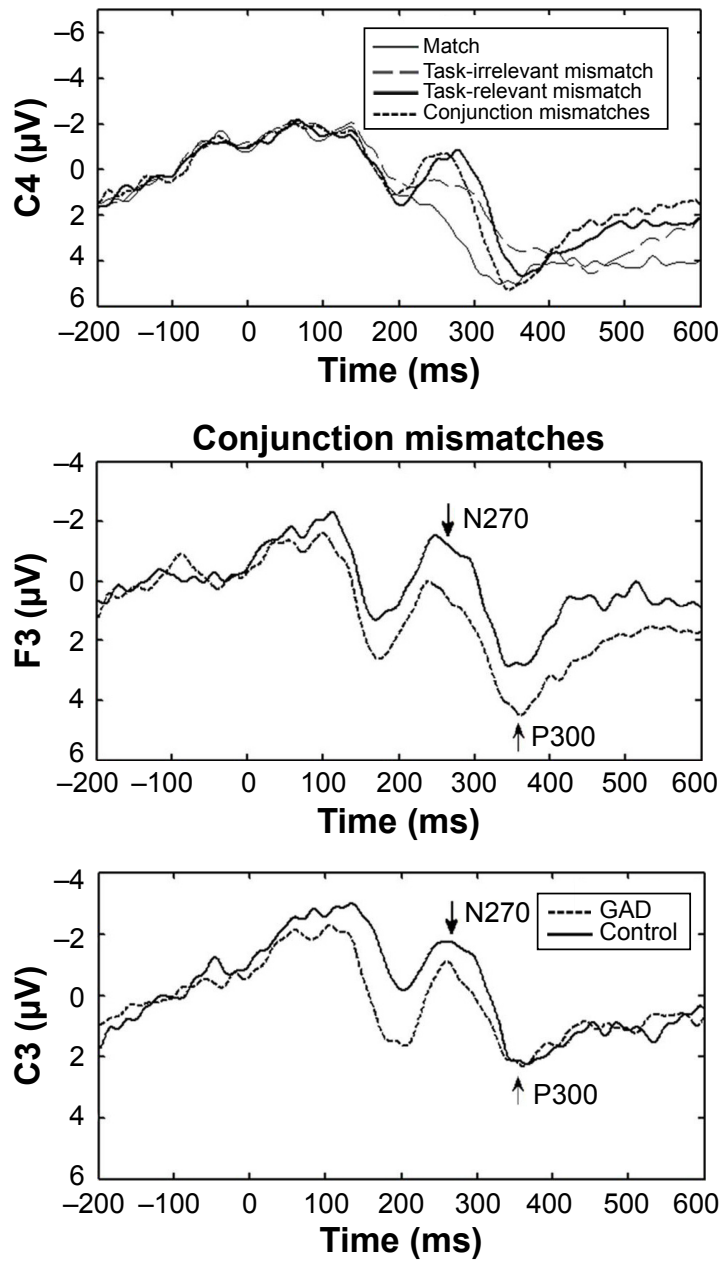

Figure I The grand-averaged ERPs for the GAD group and the control group.

Notes: Time 0 represents the onset of S2. (A) Controls: the grand-averaged ERPs for the four conditions. While the N270 was not observed in the match condition, it was recorded in the other three mismatch conditions. (B) Patients with GAD: in task-relevant mismatch condition, there was a smaller N270 amplitude at electrode F3 compared to the controls. In the conjunction mismatches condition, there was a smaller N270 amplitude at electrodes F3 and C3 compared to the controls.

Abbreviations: ERPs, event-related potentials; GAD, generalized anxiety disorder.

score were computed across all the subjects. In the taskrelevant mismatch condition, there was a significant positive correlation between scores on all three scales with the N270 amplitude at electrode F3 only: HAMA $(r=0.417, P<0.05)$, HAMD ( $r=0.431, P<0.05)$, and STAI $(r=0.381, P<0.05)$. In the conjunction mismatches condition, significant positive correlations were found at electrodes F3 and C3: F3, HAMA $(r=0.426, P<0.05)$, HAMD $(r=0.416, P<0.05)$, and STAI $(r=0.413, P<0.05)$; C3, HAMA $(r=0.458, P<0.05)$, HAMD $(r=0.448, P<0.05)$, and STAI $(r=0.446, P<0.05)$. Summarizing these findings, greater anxiety and depression scores were associated with the smaller N270 amplitude.

\section{Discussion}

Consistent with our hypotheses, this study demonstrated the attenuated N270 amplitude in individuals with GAD, 
Table 3 The mean amplitude of N270 for the two groups in each of the four conditions and at each of the chosen electrodes (mean \pm SD)

\begin{tabular}{|c|c|c|c|c|}
\hline Amplitude $(\mu \mathrm{V})$ & Match & Task-irrelevant mismatch & Task-relevant mismatch & Conjunction mismatches \\
\hline \multicolumn{5}{|l|}{ Controls } \\
\hline F3 & $1.69 \pm 2.87$ & $0.48 \pm 1.98^{\mathrm{a}}$ & $-0.46 \pm 1.92^{\mathrm{a}}$ & $-0.73 \pm 2.1 I^{\mathrm{a}}$ \\
\hline $\mathrm{F} 4$ & $2.93 \pm 3.85$ & $1.01 \pm 2.60^{\mathrm{a}}$ & $0.19 \pm 1.62^{\mathrm{a}}$ & $-0.7 I \pm I .94^{\mathrm{a}-\mathrm{c}}$ \\
\hline $\mathrm{C} 3$ & $0.96 \pm 2.91$ & $-0.61 \pm 1.58^{\mathrm{a}}$ & $-0.48 \pm I .43$ & $-1.54 \pm 1.42^{\mathrm{a}, \mathrm{c}}$ \\
\hline $\mathrm{C} 4$ & $2.8 \mathrm{I} \pm 3.49$ & $0.39 \pm 3.14^{\mathrm{a}}$ & $-0.32 \pm 1.66^{\mathrm{a}}$ & $-0.3 \mathrm{I} \pm 2.78^{\mathrm{a}}$ \\
\hline \multicolumn{5}{|l|}{ GAD } \\
\hline F3 & $2.32 \pm 2.31$ & $0.34 \pm 2.28^{\mathrm{a}}$ & $1.33 \pm 1.73^{\mathrm{d}}$ & $1.12 \pm 1.63^{\mathrm{a}, \mathrm{d}}$ \\
\hline F4 & $1.93 \pm 2.44$ & $0.91 \pm 1.74^{a}$ & $0.48 \pm 1.97^{\mathrm{a}}$ & $0.56 \pm 2.15^{\mathrm{a}}$ \\
\hline $\mathrm{C} 3$ & $1.39 \pm 2.94$ & $0.45 \pm 2.80$ & $-0.18 \pm 1.70^{\mathrm{a}}$ & $0.30 \pm 2.07^{d}$ \\
\hline $\mathrm{C} 4$ & $1.56 \pm 2.72$ & $0.83 \pm 1.80$ & $0.34 \pm 1.44$ & $0.48 \pm 2.37$ \\
\hline
\end{tabular}

Notes: ${ }^{2}$ Significantly different from the match condition in each group $(P<0.05)$. ${ }^{b}$ Significantly different from the task-irrelevant mismatch condition in each group $(P<0.05)$. 'Significantly different from the task-relevant mismatch condition in each group $(P<0.05)$. ${ }^{\circledR}$ Significantly different from the control group $(P<0.01)$. Controls are healthy subjects.

Abbreviations: SD, standard deviation; GAD, generalized anxiety disorder.

providing powerful evidence that GAD is associated with cognitive impairment. Moreover, the reduction in N270 amplitude was most pronounced in patients having GAD with greater symptoms of anxiety and depression.

This study revealed that compared to control subjects, patients with GAD displayed a smaller amplitude of N270 in response to task-relevant and conjunction mismatches, but not to task-irrelevant mismatch. Since the N270 putatively indexes selective attention, ${ }^{13}$ GAD may be associated with impaired selective attention processes that require enhanced target-stimulus processing. Our findings are consistent with the hypothesis that anxiety impairs goal-directed attention systems, even in the absence of threat-related stimuli. ${ }^{21}$ Many studies have suggested that patients with GAD suffer from a decrement in target-stimulus processing caused by an attentional bias toward emotional distracters. There is very few research using neutral stimuli. Prior to our study, it was unclear whether GAD-related deficits in selective attention were due to the emotional component of the tasks used or general deficits. The neutral stimuli are not the priority of goal-directed attention of young adults because they generally display negativity bias in cognitive processing. Therefore, it would be easier to find deficits associated with GAD using neutral stimuli than using negatively valenced ones. Our study using neutral stimuli suggests that the decreased goal-directed attention associated with GAD is a general deficit, regardless of emotional content.

Normal working memory is crucial for successful performance in the delayed matching-to-sample task. ERP abnormalities found during the present task suggest GADassociated working memory impairments. Though GAD working memory deficits have been previously reported using neuropsychological assessments, ${ }^{3,5}$ our results provide electrophysiological support for these working memory decrements in subjects with GAD.

It is worth noting that the smaller amplitude of the N270 in subjects with GAD was only observed in the left hemisphere. While the left hemisphere is thought to actively process positive emotions or approach-related behavior, the right is thought to process negative emotions or withdrawal behavior. ${ }^{22}$ Our data indicate a hemispheric asymmetry of cognitive dysfunction in subjects with GAD. Interestingly, GAD has

Table 4 The N270 peak latency of the two groups in mismatch conditions (mean \pm SD)

\begin{tabular}{llll}
\hline Latency $(\mathbf{m s})$ & Task-irrelevant mismatch & Task-relevant mismatch & Conjunction mismatches \\
\hline Controls & & & $277.27 \pm 10.22$ \\
F3 & $280.00 \pm 12.98$ & $280.93 \pm 12.57$ & $274.07 \pm 8.89$ \\
F4 & $272.87 \pm 13.24$ & $277.73 \pm 11.76$ & $278.47 \pm 11.83$ \\
C3 & $278.13 \pm 13.72$ & $282.20 \pm 17.11$ & $274.27 \pm 10.38$ \\
C4 & $274.00 \pm 13.78$ & $276.73 \pm 9.89$ & \\
GAD & & & $272.31 \pm 20.55$ \\
F3 & $270.00 \pm 17.34$ & $273.08 \pm 17.27$ & $265.92 \pm 18.40$ \\
F4 & $267.23 \pm 17.60$ & $270.46 \pm 18.04$ & $276.69 \pm 16.91$ \\
C3 & $273.92 \pm 15.95$ & $273.62 \pm 14.86$ & $267.46 \pm 17.34$ \\
C4 & $268.38 \pm 14.00$ & $271.77 \pm 16.57$ & \\
\hline
\end{tabular}

Note: Controls are healthy subjects.

Abbreviations: SD, standard deviation; GAD, generalized anxiety disorder. 
Table 5 The mean amplitude and the peak latency of the P300 for the two groups in the match condition (mean \pm SD)

\begin{tabular}{ccc}
\hline & Controls & GAD \\
\hline Amplitude $(\mu \mathrm{V})$ & & \\
P3 & $2.97 \pm 5.31$ & $1.42 \pm 3.97$ \\
P4 & $4.10 \pm 5.53$ & $2.82 \pm 3.77$ \\
Latency $(\mathrm{ms})$ & & \\
P3 & $334.20 \pm 19.43$ & $321.07 \pm 48.6 \mathrm{I}$ \\
P4 & $339.93 \pm 20.56$ & $326.60 \pm 35.91$ \\
\hline
\end{tabular}

Note: Controls are healthy subjects.

Abbreviations: SD, standard deviation; GAD, generalized anxiety disorder.

been related to increased right-hemispherical activation, especially in emotional processing. ${ }^{16}$ Our study suggests that hypoactivity of the left hemisphere in the processing of neutral information may also be a core feature of GAD. Considering that N270 is related to prefrontal activity, ${ }^{14,15}$ the hemispheric asymmetry of the $\mathrm{N} 270$ response may indicate left prefrontal hypoactivation during information processing in patients with GAD. The hemispheric differences described may open up new avenues for neuromodulation treatment of GAD that selectively targets the left prefrontal cortex.

Despite the decreased N270 amplitude in the GAD group, no behavioral differences were observed between the two groups. Electrophysiological findings in the absence of behavioral differences may be due to, on one hand, the higher sensitivity of ERPs compared to behavioral data. ${ }^{23}$ On the other hand, Etkin and Schatzberg ${ }^{24}$ suggest that intact behavioral performance can be seen in individuals with GAD primarily due to compensatory processes. Compensatory processes influencing mismatch perception require further investigation.

In this study, the peak latency and amplitude of P300 of the patients with GAD and controls were not significantly different. In line with previous studies, ${ }^{10,25}$ N270 is more sensitive than P300 in detecting minor cognitive impairment, particularly selective attention and working memory.

It is also important to note the limitations of this study. First, the small sample size might have limited statistical power to detect all but large effects. Second, patients with severe anxiety were not included in the study because they may have experienced great difficulty sitting still and remaining focused on the task. The smaller N270 amplitude may be more obvious in patients with more serious symptoms and may not be confined to the left hemisphere. This therefore requires further investigation.

In conclusion, our findings indicate that an electrophysiological marker of mismatch perception, the N270, is diminished in individuals with GAD relative to demographically similar control subjects when performing a cognitive task with no emotional stimuli. The results strongly suggest that GAD is associated with cognitive impairment, notably selective attention and working memory. Identifying such impairments will be useful for making triage and treatment decisions in subjects with GAD.

\section{Acknowledgments}

This research was supported in part by the National Natural Science Foundation of China (No 81271494, 81301138, 61301042) and Hundred Talents Program of CAS. The authors are grateful to Baoquan Min, Fengying Jiang, Yue Hou, and Yan Ding for their assistance with data collection.

\section{Disclosure}

The authors report no conflicts of interest in this work.

\section{References}

1. Muller J, Roberts JE. Memory and attention in obsessive-compulsive disorder: a review. J Anxiety Disord. 2005;19(1):1-28.

2. Johnsen GE, Asbjornsen AE. Consistent impaired verbal memory in PTSD: a meta-analysis. J Affect Disord. 2008;111(1):74-82.

3. Tempesta D, Mazza M, Serroni N, et al. Neuropsychological functioning in young subjects with generalized anxiety disorder with and without pharmacotherapy. Prog Neuropsychopharmacol Biol Psychiatry. 2013; 45(1):236-241.

4. Gualtieri CT, Morgan DW. The frequency of cognitive impairment in patients with anxiety, depression, and bipolar disorder: an unaccounted source of variance in clinical trials. J Clin Psychiatry. 2008; 69(7):1122-1130

5. Butters MA, Bhalla RK, Andreescu C, et al. Changes in neuropsychological functioning following treatment for late-life generalized anxiety disorder. Br J Psychiatry. 2011;199(3):211-218.

6. Airaksinen E, Larsson M, Forsell Y. Neuropsychological functions in anxiety disorders in population-based samples: evidence of episodic memory dysfunction. J Psychiatr Res. 2005;39(2):207-214.

7. Castaneda AE, Suvisaari J, Marttunen M, et al. Cognitive functioning in a population-based sample of young adults with anxiety disorders. Eur Psychiatry. 2011;26(6):346-353.

8. Wang H, Wang Y, Wang D, Cui L, Tian S, Zhang Y. Cognitive impairment in Parkinson's disease revealed by event-related potential N270. J Neurol Sci. 2002;194(1):49-53.

9. Mao W, Wang Y, Wang D. Cognitive impairment in major depressive disorder revealed by event-related potential N270. Clin EEG Neurosci. 2005;36(1):9-14.

10. Mao W, Yang J, Wang M, et al. Event-related potential N270 in detecting cognitive impairment in patients with transient ischemic attack. J Clin Neurophysiol. 2006;23(6):559-564.

11. Folstein JR, Van Petten C. Influence of cognitive control and mismatch on the N2 component of the ERP: a review. Psychophysiology. 2008; 45(1):152-170.

12. Zhang X, Wang Y, Li S, Wang L, Tian S. Distinctive conflict processes associated with different stimulus presentation patterns: an event-related potential study. Exp Brain Res. 2005;162(4):503-508.

13. Mao W, Wang Y. Various conflicts from ventral and dorsal streams are sequentially processed in a common system. Exp Brain Res. 2007; 177(1):113-121.

14. Zhang X, Ma L, Li S, Wang Y, Weng X, Wang L. A mismatch process in brief delayed matching-to-sample task: an fMRI study. Exp Brain Res. 2008;186(2):335-341. 
15. Bocquillon P, Bourriez JL, Palmero-Soler E, Molaee-Ardekani B, Derambure P, Dujardin K. The spatiotemporal dynamics of early attention processes: a high-resolution electroencephalographic study of N2 subcomponent sources. Neuroscience. 2014;271(1):9-22.

16. Hilbert K, Lueken U, Beesdo-Baum K. Neural structures, functioning and connectivity in generalized anxiety disorder and interaction with neuroendocrine systems: a systematic review. J Affect Disord. 2014; $158(1): 114-126$

17. Hamilton M. The assessment of anxiety states by rating. $\mathrm{Br} J \mathrm{Med}$ Psychol. 1959;32(1):50-55.

18. Hamilton M. A rating scale for depression. J Neurol Neurosurg Psychiatry. 1960;23(1):56-62.

19. Spielberger CD, Gorsuch RL, Lushene R, Vagg PR, Jacobs GA. Manual for the State-Trait Anxiety Inventory (STAI). Palo Alto, CA: Consulting Psychologists Press; 1983.

20. Wang Y, Cui L, Wang H, Tian S, Zhang X. The sequential processing of visual feature conjunction mismatches in the human brain. Psychophysiology. 2004;41(1):21-29.
21. Eysenck MW, Derakshan N, Santos R, Calvo MG. Anxiety and cognitive performance: attentional control theory. Emotion. 2007;7(2):336-353.

22. Trinkl M, Greimel E, Bartling J, Grunewald B, Schulte-Korne G, Grossheinrich N. Right-lateralization of N2-amplitudes in depressive adolescents: an emotional go/no-go study. J Child Psychol Psychiatry. 2015;56(1):76-86.

23. Wilkinson D, Halligan P. The relevance of behavioural measures for functional-imaging studies of cognition. Nat Rev Neurosci. 2004;5(1):67-73.

24. Etkin A, Schatzberg AF. Common abnormalities and disorder-specific compensation during implicit regulation of emotional processing in generalized anxiety and major depressive disorders. Am J Psychiatry. 2011;168(9):968-978.

25. Sun W, Wang W, Wu X, Wang Y. Antiepileptic drugs and the significance of event-related potentials. J Clin Neurophysiol. 2007;24(3) $271-276$.
Neuropsychiatric Disease and Treatment

\section{Publish your work in this journal}

Neuropsychiatric Disease and Treatment is an international, peerreviewed journal of clinical therapeutics and pharmacology focusing on concise rapid reporting of clinical or pre-clinical studies on a range of neuropsychiatric and neurological disorders. This journal is indexed on PubMed Central, the 'PsycINFO' database and CAS,

\section{Dovepress}

and is the official journal of The International Neuropsychiatric Association (INA). The manuscript management system is completely online and includes a very quick and fair peer-review system, which is all easy to use. Visit http://www.dovepress.com/testimonials.php to read real quotes from published authors.

Submit your manuscript here: http://www.dovepress.com/neuropsychiatric-disease-and-treatment-journal 